\title{
Speech Files Compression Based on Signal Feature
}

Khalil I. Alsaif

Khalil_alsaif@hotmail.com

Computer center

University of Mosul, Iraq

Received on: 03/05/2006
Saja J. Mohammed

Sj_alkado@uomosul.edu.iq

College of Computer Science and Mathematics

University of Mosul, Iraq

Accepted on: 05/03/2007

\section{ABSTRACT}

In this research a new algorithm was suggested for compressing speech files added a new style for storing signals, The suggested idea of compression begins with recording the speech via the microphone, then starting the proposed processing steps as follows :

- Removing silent period.

- Select the number of resulted signal samples.

- Segmenting the resulting signal to number of frames.

- Applying one of the curves fitting algorithms and obtaining the coefficients for the mathematical representation.

- Storing the results in a new file format with .ssc (Speech Signal Compression) extension.

While the decompression process consisted of the reversal compression process steps, the signal is reconstructed using curve fitting coefficients which were stored in the new file, followed by returning the selected sample, then returning the silent period to their original location and finally listening to the retrieved speech signal. When the proposed algorithm had been applied on the files with different speech contents, the compression ratio was approximately (16.283\%), and the ratio of SEGSNR was approximately (25.195dB).

Keywords: compression, speech signal, feature extraction, Silent Period

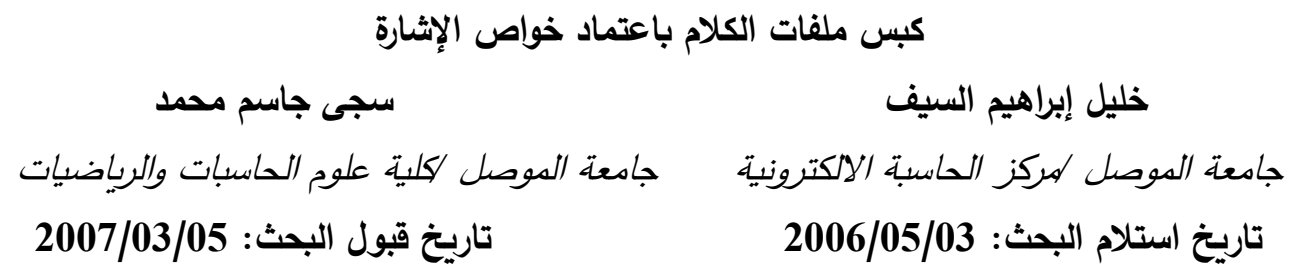




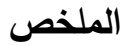

أُقترح في البحث خوارزمية لعملية كبس ملفات الكلام أضفت طابعاً آخر على أسـاليب

خزن إثارة الكلام، تبدأ فكرة الكبس المقترحة باكتساب الكلام من لاقط الصوت، ثم البدء بخطوات المعالجة المقترحة والتي تضمنت:

• العمل على إزالة فترات الصمت (Silent Period).

• اقتباس بعض العينات للإثارة الناتجة.

• تقطيع الإثارة الناتجة ومن ثم تطبيق إحدى طرائق مواءمة منحني الإشارة (Curve Fitting)

$$
\text { والحصول على معاملات التمثيل الرياضي للإثارة. }
$$

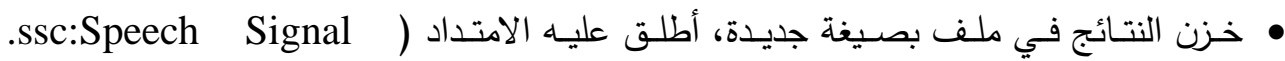

.(Compression

أما عملية فك الكبس فقد تضمنت خطوات معاكسة لعملية الكبس إذ يتم إعادة بناء كائنات

الإشارة من جديد باستخدام معاملات مواءمة المنحني(Curve Fitting Coefficients) المخزونـة في الملف الجديد، تتبعها إعادة العينات المقتطعة من الإشارة ثم إرجاع فترات الصمت إلى مواقعها

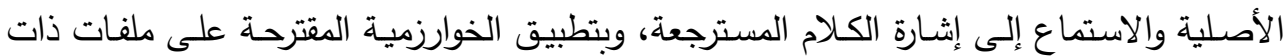

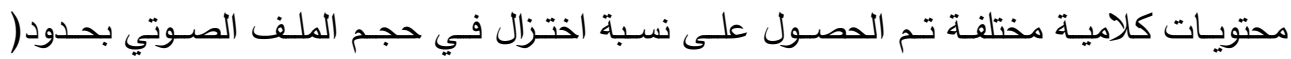

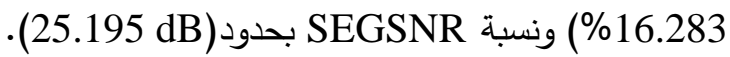
الكلمات المفتاحية: الكبس، اشارة الكلام، استخلاص الخواص، فتردات فلات الصدمت. 1

لا يخفى على أحد أهمية الدور الذي تؤديه وسـائل الاتصـال الخاصـة بالكلام في الحياة

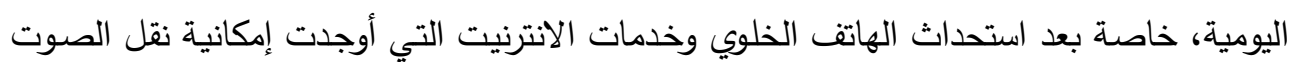

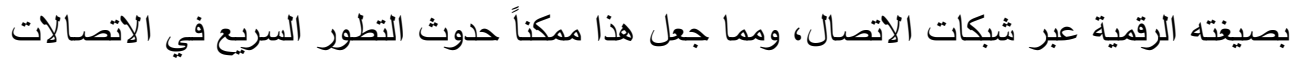

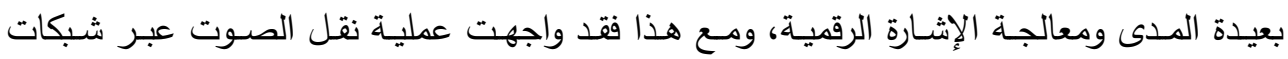

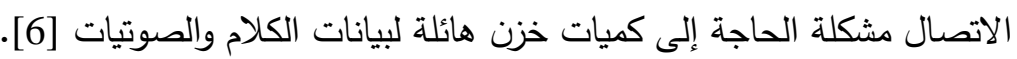

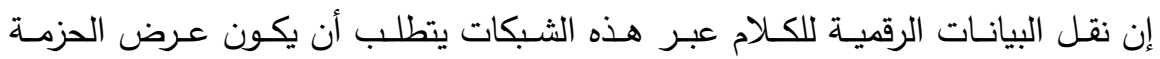
المستخدمة لنقل البيانات بقدر كافٍ بحيث تتقل البيانات بنوعية جيدة ومقبولة تؤدي الغرض من هن

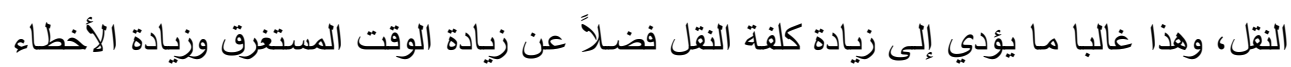


المتوقعة أثناء نقل البيانات. عليه فقد أصبحت الحاجة ملحة إلى وجود طرائق تعمل على خزن إثارة الكلام بأقل حجم ممكن واستحداث خوارزميات خاصة تعمل على كبس هذه البيانات، لأجل التقليل من كمية الخزن المطلوبة للبيانات ومن ثم التقليل من عرض الحزمة المطلوبة لنقلها وزيادة سرعة النقل وتقليل احتمالية حدوث الأخطاء أثناء عملية النقل [8].

$$
\text { 2- 20 - 2 الصوت والكلام: }
$$

Speech يوصف الكلام بأنه يمثل الصوت الناتج من جهاز التكلم الخاص بالإنسان (Production System

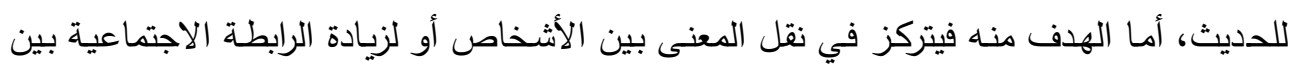

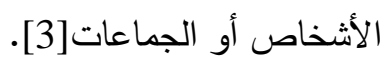

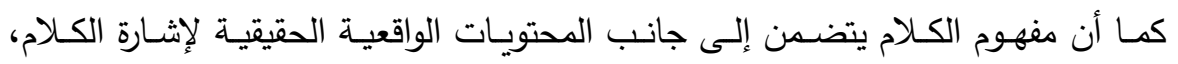

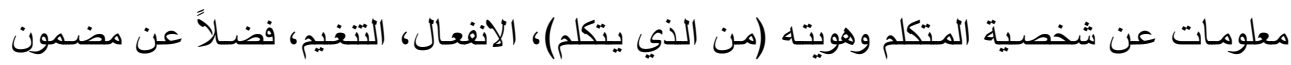
الرسالة المنقولة بالكلام، كل هذه العوامل مهمة بالنسبة إلى مفهوم الكلام [3].

$$
3 \text { - كبس البيانات بين المحاسن والمساوئ: }
$$

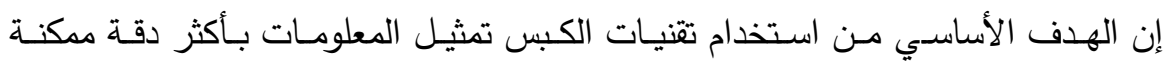

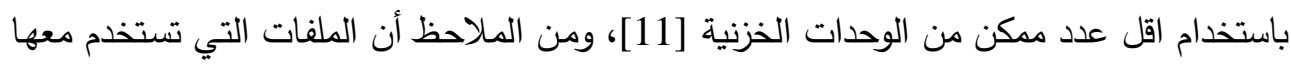
تقنيات الكبس تمتاز بما ياتتي[1][2]: •صغر حجم الملف نسبة إلى حجم الملف الأصلي، وهذا ما يجعله يشغل حيزاً اقل في الذاكرة.

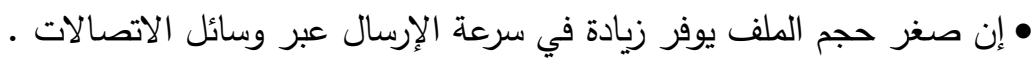
• •تميز الملفات الدكبوسة بوجود نوع من أنواع ترميز البيانات (Data Coding) التي توفر أمنية للملف بوصفه نتيجة عرضية لعملية الكبس. • عند إرسال أي ملف مكبوس فان عرض الحزمة (Bandwidth) المطلوبة لإرسال ذلك الملف تكون اقل من حالة كونه غير مكبوس.

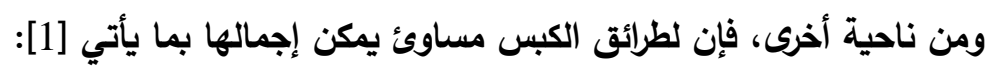


• عند نقل بيانات ملف مكبوس فإن حدوث الأخطاء أثناء عملية النقل تؤثر تأثيراً كبيراً على الملف المكبوس نسبة إلى الملف غير المكبوس، ذلك أنها قد تؤدي إلى تغيير أو فقدان المعلومـات الضرورية التي تم الاحتفاظ بها عند عملية الكبس. • تحتاج الملفات إلى تقنيات خاصـة لغرض كبسها مما يؤدي إلى إضـافة خوارزميات أخرى إلى

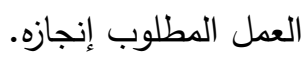

• ضياع بعض المعلومات التي قد تكون مهمة للغاية ولاسيما عند استخدام طرائق الكبس بفقدان

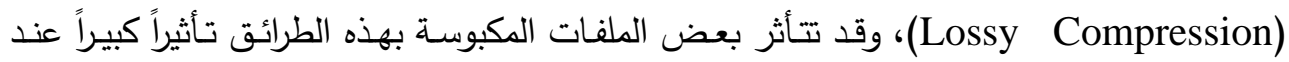
استرجاعها فتصبح الملفات المسترجعة ذات معلومات مشوشة(Noise).

$$
4
$$

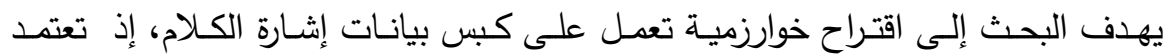

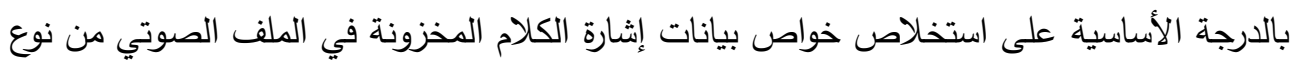

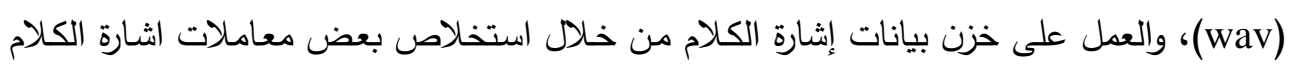
بحيث تؤدي إلى اختزال جزء كبير من حجم البيانات على ذاكرة الحاسوب، وتوفير إمكانية إعادة

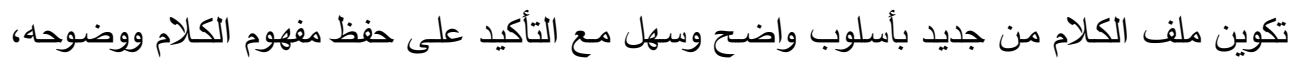
والعمل قدر الإمكان على تقليل الضوضاء الناتجة عن معالجة إثارة الكلام.

\section{5- نظرة تاريخية إلى معالجة الكلام:}

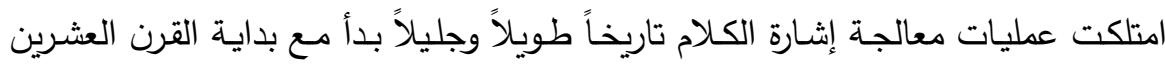

وامتد إلى بدايـة القرن الحادي والعشرين، فقد بدأ العمل على كبس إثـارة الكلام منذ عام 1928 عندما قدمت مهندسة الهواتف هومر دادلي (Homer Dudley) فكرتها التي تحدثت عن كبس

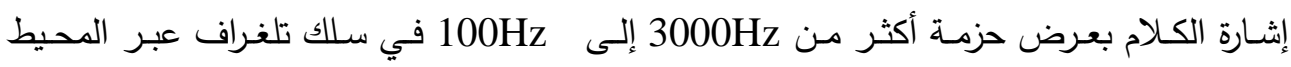
الأطلسي، إذ نصت فكرة هومر على ترميز الإثارة ثم نقلها، فبدلا من نقل إثارة الكلام نفسها يكفي إرسال وصف عن تلك الإشارة بحيث يمكن إعادة تكوينها من جديد عند الجهة المستلمة[9]. 
من جهة أخرى بدأ في الأربعينات العمل على كبس إثـارة الكلام اعتماداً على ترميز صيغة الموجة(Waveform Coding) بطريقة تحميل الدلتا(Delta-Modulation) وذلك في بحوث شركة فليبس الهولندية.

PCM: ) وبعد أن اشتهرت عملية خزن الإثارة الصوتية بطريقة التحميل برمز الإثشارة

(Pulse Code Modulation ADPCM: Adaptive Differential Pulse Code ( بالفرق التكيفي لرمز الإثارة (Modulation

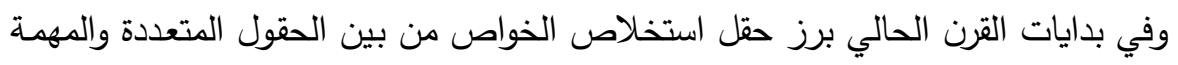

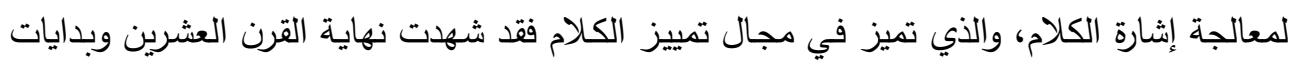
القرن الواحد والعشرين تطويراً للعديد من طرائق استخلاص خواص الإثارة من بين هذه البحوث

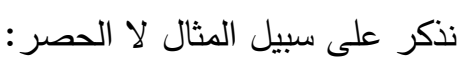
قام كل من(جونك هوان لي و هايونك مين بارك و هويونك جنك و سويونك لي) في

عام(2002)، بتقديم تقرير تحت عنوان Feature Extraction Using Independent) والذي تضمن اقتراح طريقة جديدة في مجال استخلاص الخواص Component Analysis)

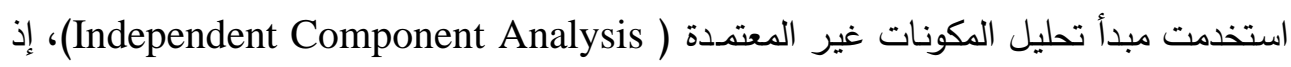
قامت هذه الطريقة بفصل كفوء لككونات الضوضاء (التي اكتسبت إلى إثارة الكلام خلال تسجيله)

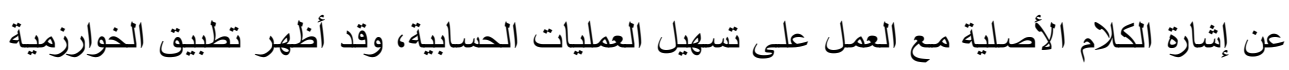
المقترحة نتائج أداء مقبولة[7] الاصلة.

وقام الباحثان (مارك سكورونسكي و جون هاريس) من جامعة فلوريدا في عام (2003)

$$
\text { بنشر بحث تحت عنوان: }
$$

\section{Improving the Filter Bank of a Classic Speech Feature Extraction algorithm}

الذي قدم طريقة جديدة لتصميم مرشح (Filter Bank) جديد يستخدم في طريقة الـ(MFCC) التي

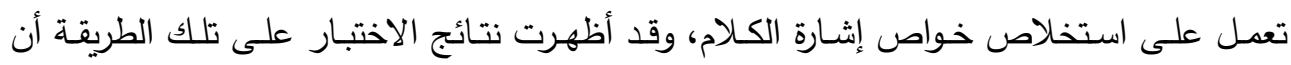

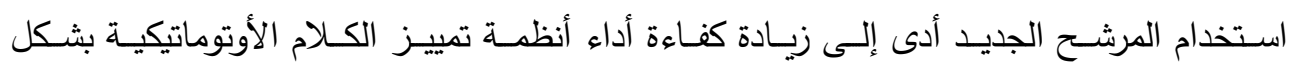
واضح[10]. 
أما من جامعة الموصل فقد قدمت الباحثة إسراء عبد السلام عبد القادر عام 2001 بحثها الموسوم (كبس الصوت عند الزمن الحقيقي) الذي استخدمت فيه خوارزمية(LZW) في عملية كبس علم الصوت قبل خزنه على ذاكرة الحاسوب والعمل على فك الكبس عند محاولة استماع الصوت مجدداً

6 - خوارزمية الكبس وفك الكبس المقترحة: اولا: مراحل عملية الكبس:

تعتمد الخوارزمية المقترحه على تمثيل الكلام المكتسب إلى الحاسوب بصيغة رقمية في مجال الزمن، ثم اختزال أجزاء منها ومن ثم إيجاد تمثيل رياضي لمعاملات الزمن المتبقية التي يتم

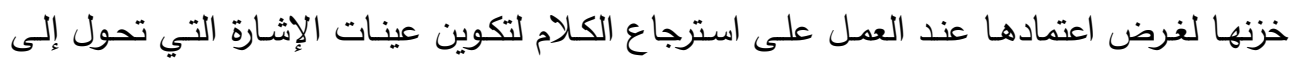
الصوت الذي تم استقباله ثم خزنه. المرحلة الأولى: تبدأ مراحل الخوارزمية المقترحة بخطوة التهيئة، إذ يتم تهيئة منطلبات العمل بتحديد القيم المطلوبـة لتتفيذ البرنـامج وقد تمت تهيئة المثال المعتمد في وصف لبـ الخوارزميـة حسب القيم الآتية: • الوقت المستغرق للتسجيل: 3 ثوانٍ. • معدل التعيان(S000KHz :Sampling Rate) • الحد الأدنى والأعلى لاتساع موجة صمت:0.03dB، 0.02dB على التوالي.

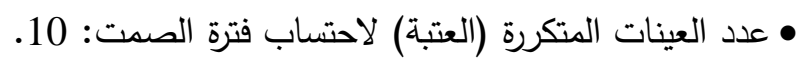
• عدد النبضات (Sample) المعتمدة في انتخاب العينات : 2.

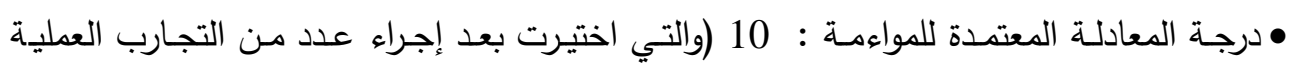
وإيجاد الأفضل) • حجم المقطع الواحد للإثارة :20 عينة لكل مقطع.

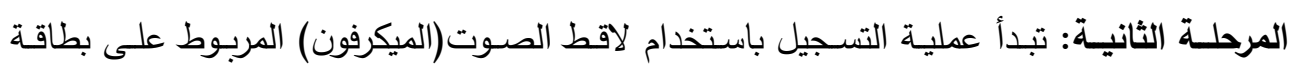
الصوت داخل الحاسوب، ومن البديهي معرفة إن حجم الإشارة بهذه الشروط يكون 24000 عينة (Sample)، الثكل(1) ناتج من رسم إثارة كلام مسجلة اعتماداً على ما تم ذكره. 


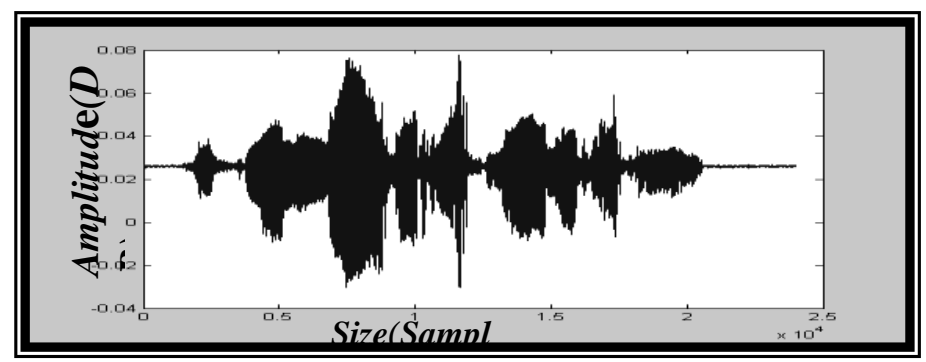

\section{الثكل(1): إثارة مسجلة بصوت رجل عند التلفظ بالبسملة}

المرحلـة الثالثة: تبدأ عمليـة خزن بيانات الإثـارة الداخلة إلى ملف مـن نوع (wav)، ثم قراءة البيانات من الملف إلى مصفوفة أحادية البعد، وتجدر الإشارة إلى أن حجم هذه المصفوفة عند خزن

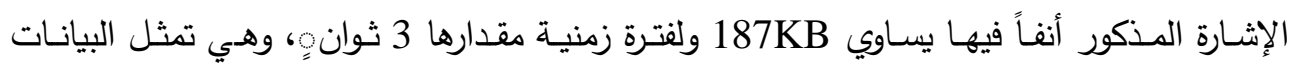
الحقيقية للكـلام، ومـن البديهي إن يزداد حجم الملف الصـوتي عند زيـادة الفترة الزمنيـة للتسـيل والعكس صحيح. المرحلة الرابعة : تبدأ في هذه المرحلة أولى خطوات المعالجة المقترحة لغرض كبس بيانات الكلام

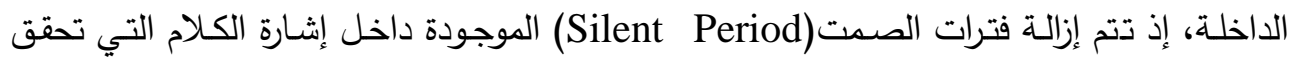

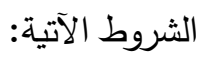
• أن تكون ضمن مدى اتساع موجة الصمت المعتمدة(الحد الأدنى والأعلى المعتمدين). • أن تساوي قيمة العتبة(عدد التكرارات) المثبتة أو تتجاوزها. مع الاحتفاظ بعناوين بداية كل فترات الصمت المقتطعة وحجمها في مصفوفة ذات بعدين

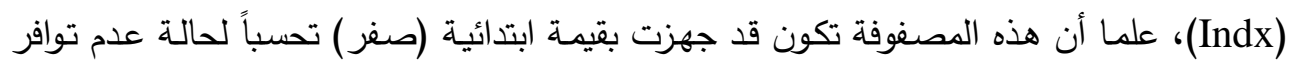
فترات الصدت في الملف.

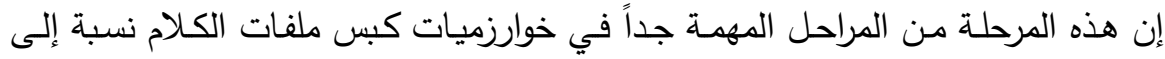
طبيعة كحلام الإنسان الذي يحوي العديد من الوقفات بين الكلمات أو العبارات أو فقرات الحديث، خاصـة إذا كان وقت الكلام طويلا نوعا مـا مثال ذلك أثناء إلقاء الخطابات أو أثناء عملية تغيير المتحدث[5].

عليه بالاعتمـاد على الإشـارة السابقة وعندما تكون قيمة العتبة (10 عينة) سيكون نـاتج إخراج هذه المرحلة المصفوفة الموضحة في الجدول(1)، والإشارة الموضحة في الثكل (2): 
الجدول(1) هيئة المصفوفة الناتجة (indx)

\begin{tabular}{|c|c|}
\hline عول| & طول فترة الصماية فترة الصمت \\
\hline 1 & 1914 \\
\hline 1918 & 33 \\
\hline 1952 & 10 \\
\hline$\vdots$ & $\vdots$ \\
\hline 20449 & 3558 \\
\hline
\end{tabular}

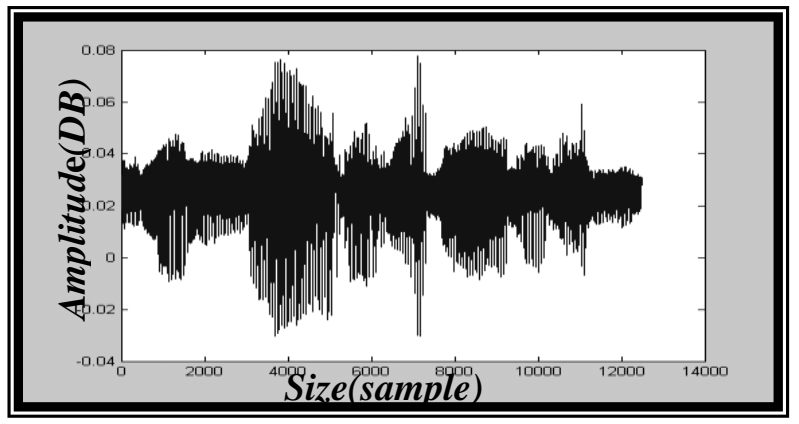

Sample|الشكل(2): الإثارة بعد إزالة فترة الصمت منها وبقيمة عتبة مقدارها

المرحلة الخامسة: يتم في هذه المرحلة اقتطاع عينات محددة من الإثارة الناتجة لغرض معالجتها

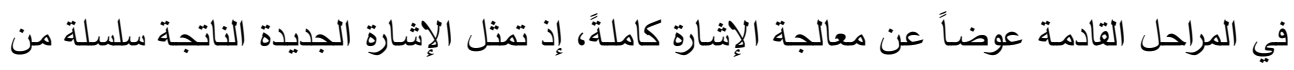
قيم جديدة كل قيمة فيها مساوية لمعدل عدد محدد من العينات الأصلية للإشارة والذي قد تم تثبيته في مرحلة التهيئة وخزنـه في المتغير الافتراضـي(Smpl)، الثكل (3) يوضـح نـاتج هذه المرحلة عندما تكون قيمة المتغير (Smpl) 


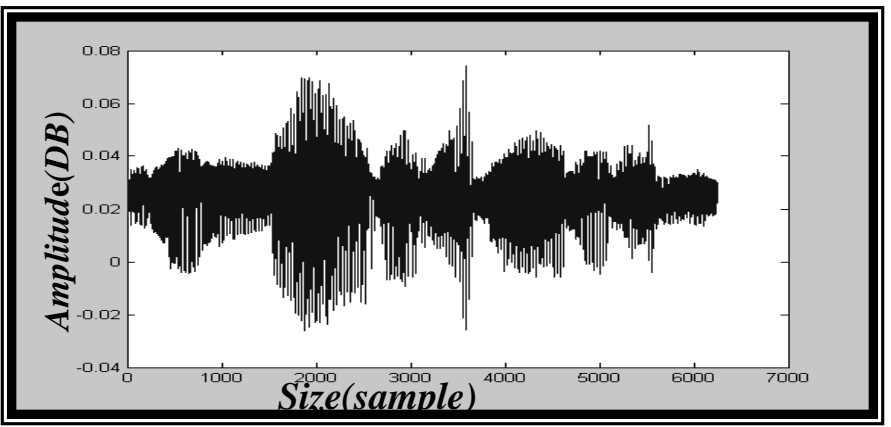

الثكل(3): الإثارة الناتجة بعد اقتطاع عينات منها

في الثكل أعلاه يمكن وصل حجم الإشارة إلى ما يقارب 6000 عينة أي قد تم فقدان ما يعادل ثلثي حجم الإثارة الأصلية، فضلاً عن كون هذه المرحلة ذات تأثير واضح في مرحلة الكبس القادمة.

ومن الجدير بالذكر أن زيادة عدد العينات المقتطعة لحساب المعدل تئدي إلى زيادة نسبة

التشوه في الإشارة الناتجة بعد عملية فك الكبس، لهذا كان اختيار قيمة المتغير (Smpl) كما هي أعلاه ناتجا عن عدد من المحاولات مع دراسة التأثير في عامل التشوه في الإشارة (SEGSNR).

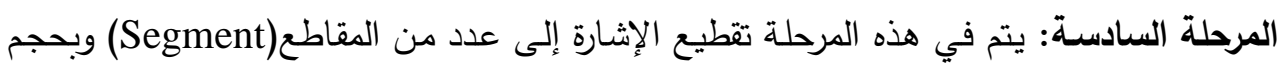
20 عينة لكل مقطع، وخزن الناتج في مصفوفة ذات بعدين. المرحلة السابعة: يتم في هذه المرحلة تطبيق طريقة مواءمة المنحنيات (Curves Fitting) لإيجاد اقرب منحني يتواءم مع منحني الإشارة الناتجة من الإشارة الأصلية وباستخدام إحدى الدوال الجاهزة في لغة (Matlab) التي استخدمت في برمجة الخوارزمية المقترحة، وتتضمن المرحلة في تطبيقها إجراء عملية كبس أخرى، فبعد عملية تقطيع الإشارة يتم اخذ كل مقطع وتطبيق أسلوب المواءمـة عليه بمعادلة من الدرجة العاشرة، أي ستكون المعادلة الناتجة كالاتي: $f(x)=a_{0}+a_{1} x+a_{2} x^{2}+a_{3} x^{3} \ldots . .+a_{9} x^{9}+a_{10} x^{10}$

$$
\begin{aligned}
& \text { إذ أن a a a : ت تمثل قيم المعاملات المطلوب إيجادها. } \\
& \text {.X تمثل قيمة العينة عند النقطة : f(x) }
\end{aligned}
$$

عليـه سـيكون نـاتج هذه المرحلـة لكل مقطع مـن الإشـارة سلسـلة مـن معـاملات مواءمـة المنحني التي يبلغ عددها 11 معاملاً، وبهذا يكون قد تم التعويض عن كل 20 عينة من الإشارة 
ب11 معاملاً، وفي هذه المرحلة يكون الملف قد فقد ما يقارب الـ45 \% من حجمه الناتج من المراحل

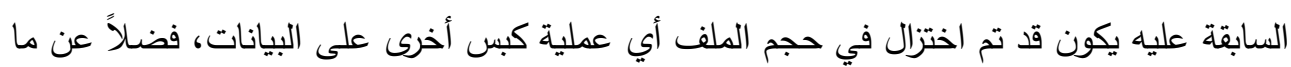

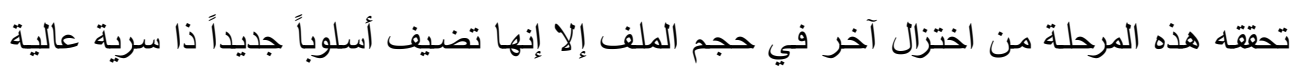
غير مطروقة في أساليب ترميز الكلام وتثفيره ، كذلك أدت إلى دعم طرائق خزن الملفات الصوتية من خلال خزن معاملات مواءمة الإثارة عوضاً عن خزن قيم الإثارة الصوتية نفسها أو باستخدام طرائق ترميز الكلام المعروفة. تُخزن نتائج هذه العملية في مصفوفة تحت اسم (noly_coeff)،وبحجم (n*11)،إذ يمثل nدد مقاطع الإشارة الكلي وكما موضح في الشكل(4).

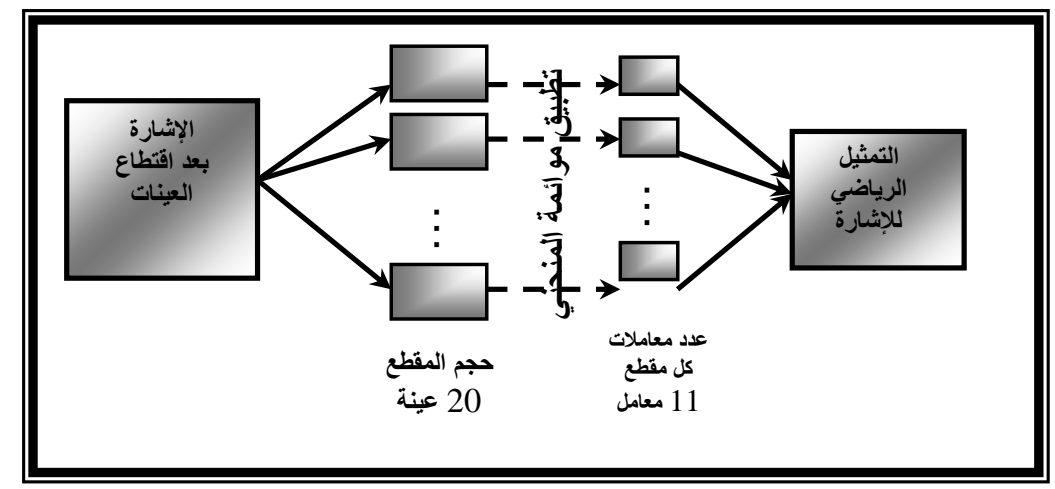

الثكل(4): تطبيق مواءمة الإشارة

المرحلة الثامنة: وتتضمن عملية خزن نتائج العمل وفق الخطوات الآتية: • ترتيب نواتج العمل المهمة في مصفوفة موحدة تضم أولاً بيانات المصفوفة (indx) التي تم فيها خزن عناوين وأطوال بدايات فترات الصمت المقتطعة في المرحلة الرابعة من مراحل العمل، تليها نواتج عملية مواءمة منحني الإشارة المخزونة في المصفوفة (poly_coeff). • إعداد القيم التي سوف تمثل بادئة الملف الجديد، كما في الجدول(2): 
(الجدول(2): بادئة الملف (SSC.)

\begin{tabular}{|c|c|}
\hline الاستخدام & حجم الحقل \\
\hline 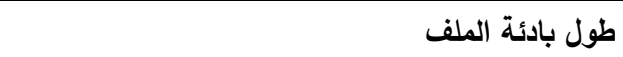 & 8Byte \\
\hline طول بيانات الصوت الحقيقية & 8Byte \\
\hline نسبة التعيان & 8Byte \\
\hline الحد الأدنى لمدى اتساع موجة الصمت & 8Byte \\
\hline الحد الأعلى لمدى اتساع موجة الصمت & 8Byte \\
\hline عدد العينات المتكررة (العتبة) لاحتساب فترة الصمت & 8Byte \\
\hline عدد فترات الصمت المقتطعة ع & 8Byte \\
\hline عدد النبضات (Sample) المعتمدة في انتخاب العينات & 8Byte \\
\hline عدد مقاطع الإثارة & 8Byte \\
\hline حجم كل مقطع & 8Byte \\
\hline عدد العينات المتبقية من تقطيع الإشارة & 8Byte \\
\hline درجة المعادلة المعتمدة للمواءمة & 8Byte \\
\hline محجوزة للاستخدام المستقبلي & 32Byte \\
\hline
\end{tabular}

• خزن نواتج الخطوتين السابقتين في ملف جديد وبصيغة خزن جديدة خاصـة بالأسلوب المقترح، وقد تم اختيار الرمز) (ssc: Speech Signal Compression) ليكون امتدادا لهيئة ملف هولف الخزن المقترح، يوضح الثكل(5) هيكلية الملف الناتج: 


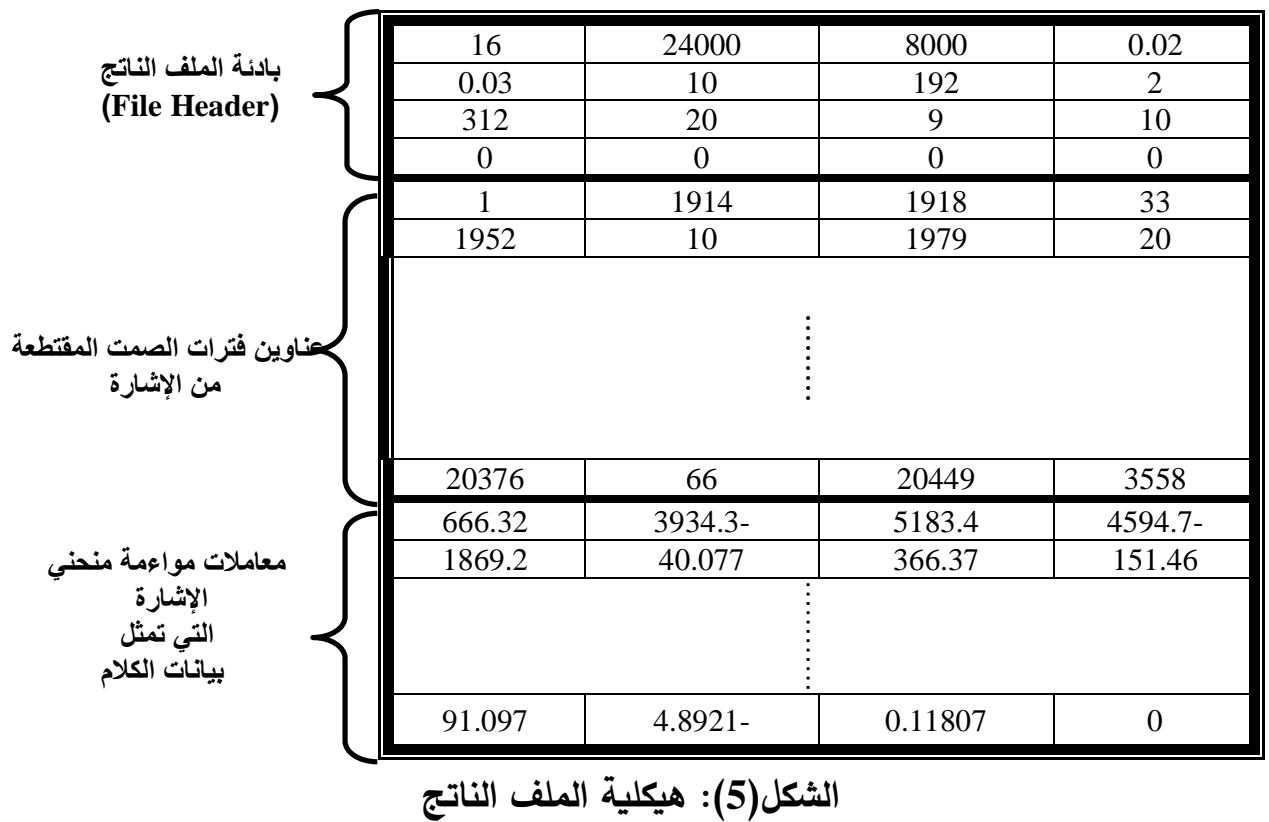

ثانيا: عملية فك الكبس:

إن خطوات خوارزمية فك الكبس تأخذ مجرى التتفيذ المعاكس لخطوات خوارزمية الكبس،

$$
\text { التي يمكن تلخيصها بما يأتي: }
$$

المرحلـة الأولـى:تبـدأ عمليـة فك الكبس بقراءة الملف المكبوس من نوع (ssc. والحصـول على

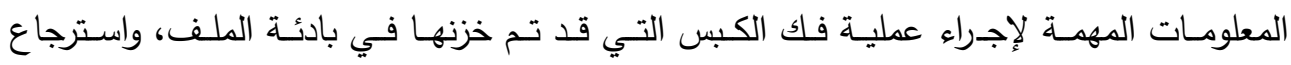
المصفوفتين (indx) و (poly_coeff) من الملف بأبعادهما الأصلية.

المرحلـة الثانيـة: تتم إعـادة بنـاء الإشـارة واسترجاع مكوناتها باستخدام معاملات مواءمـة المنحني

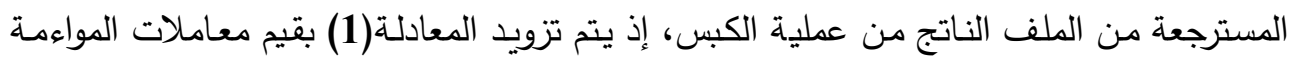

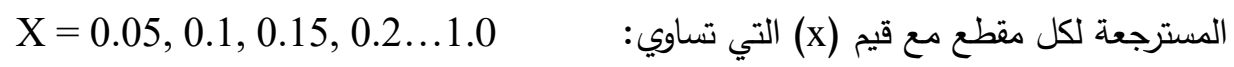


المرحلة الثالثة:تجميع المقاطع المسترجعة من المرحلة السابقة وخزنها في مصفوفة ذات بعد أحادي لأغراض المعالجة اللاحقة لفك الكبس، يوضـح الثكل (6) الإثـارة الناتجـة من عملية إعادة بنـاء

الإشارة وتجميعها :

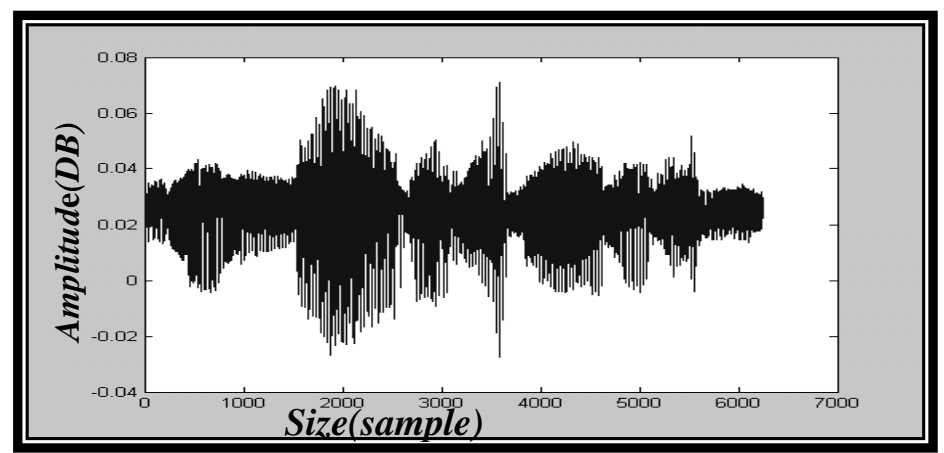

الثكل(6): الإشارة بعد إعادة بنائها وتجميعها

المرحلة الرابعة: استرجاع العينات التي تم اختزالها من الإشارة من خلال تكرار كل قيمة عينة

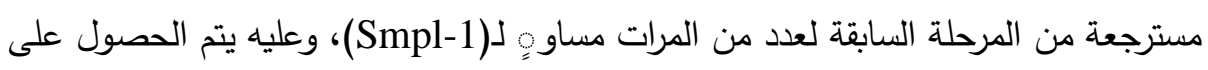

الإشارة الموضحة في الشكل (7).

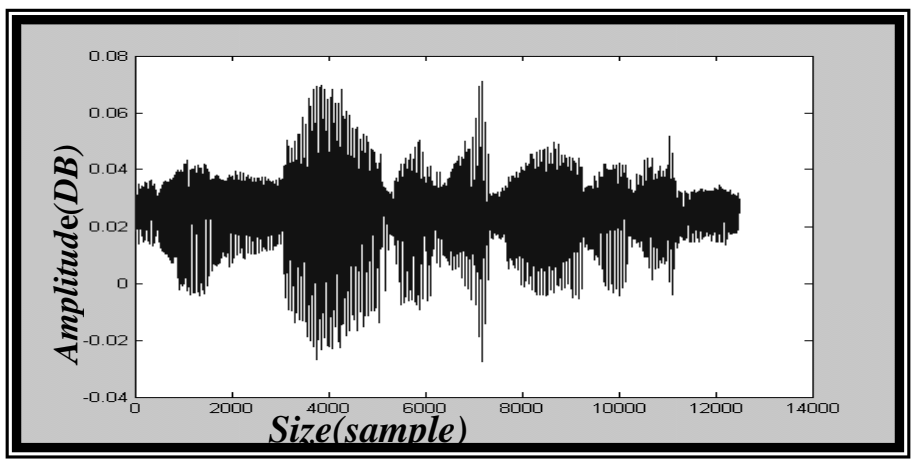

الثكل(7): استرجاع العينات المقتطعة من الإثارة

المرحلة الخامسة: تبدأ عملية إرجاع فترات الصمت المقتطعة إلى الإثارة، وتتم الاستعانة بشكل كلي

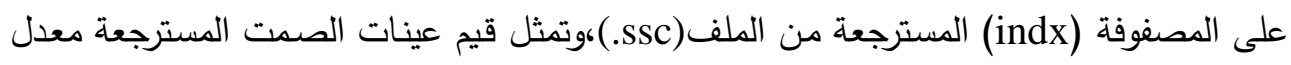


القيمتين الدنيا والعليا لاتساع موجة الصمت في الإشـارة الأصلية اللتين قد تم تثبيتهـا في بادئة الملف المستحدث.

المرحلة السادسة: بعد تتفيذ المراحل أعلاه يكون قد تم الحصول على الإشارة النهائية المسترجعة

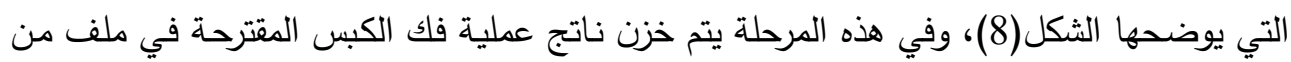
نوع (wav) ثم إعادة تشغيل الملف واستماع إثارة الكلام الناتجة.

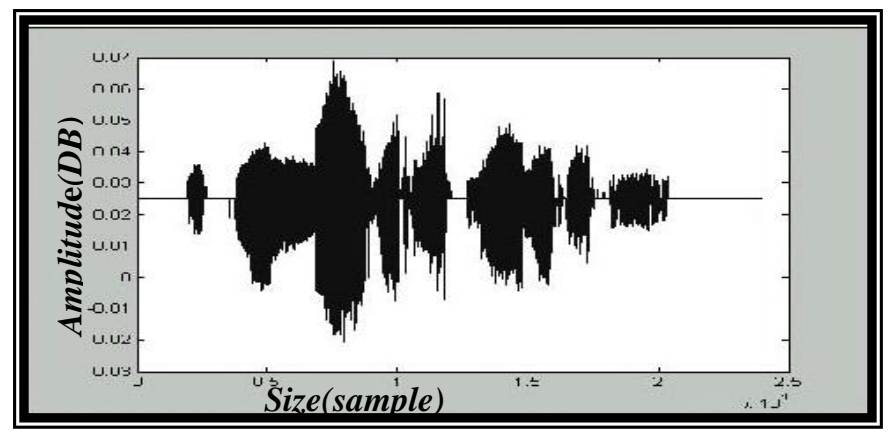

الثكل(8): الإثارة المسترجعة بعد إعادة فترات الصمت

وعند مقارنـة النتائج من خـلال رسم الإثـارتين الأصلية والمسترجعة كلتيهما يتبين مقدار

التقارب العالي بين شكلي الإشارتين، وكما هو موضح في الثكل(9) إذ يمثل الرسم الأول الإشارة الأصلية المسجلة للكلام، أما الرسم الثاني فيمثل الإشارة المسترجعة من عملية الكبس وفك الكبس.

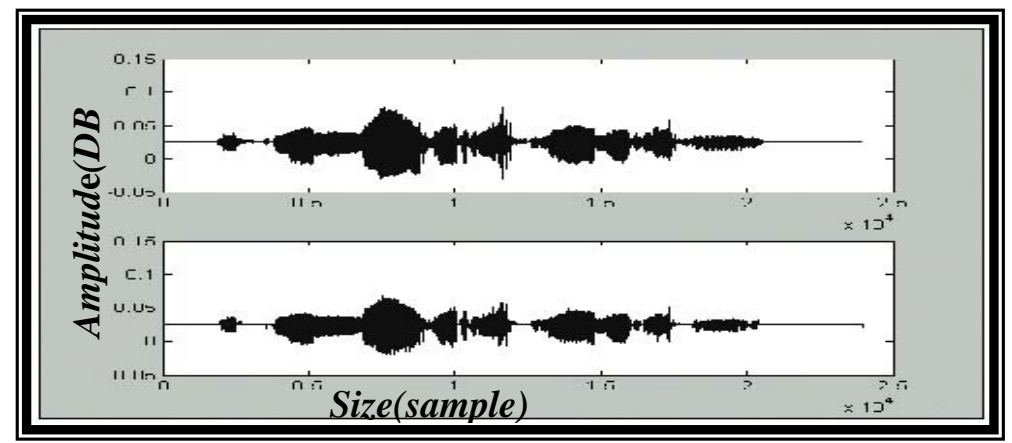

الشكل(9): الإثارة الأصلية والإثارة المسترجعة من عملية الكبس وفك الكبس

المخطط الصندوقي للخوارزمية المقترحة: 

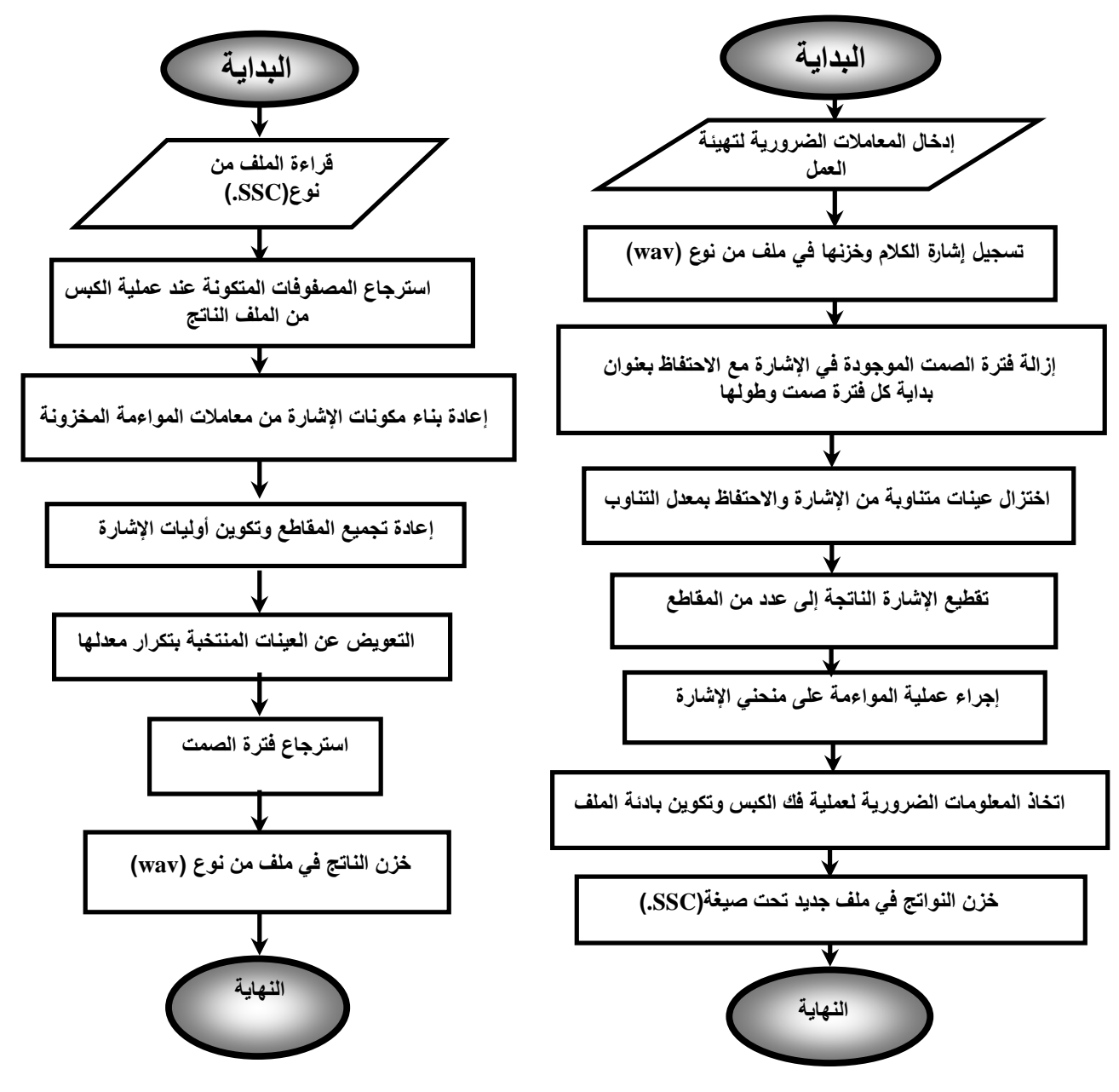

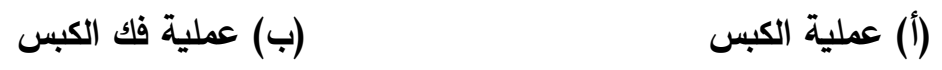
الثكل(10) المخطط الصندوقى للخوارزمية المقترحة

7- مناقثة النتائج : تمت دراسة مدى كفاءة الخوارزمية المقترحة على ملفات مختلفة وذلك باعتماد الاختبارات الآتية التي تعمل على دراسة نسبة الكبس وجودة الكلام المسترجع: 
أولا: اختبار مدى كفاءة الكبس:

من المعروف إن قياس مدى كفاءة أي خوارزمية للكبس يعرف من خـلال حساب نسبة

الكبس من المعادلة (2).

نسبة الكبس= (حجم الملف بعد الكبس /حجم الملف قبل الكبس)* 100\% ..... (2) عليه تكون نسبة الكبس الناتجة من تطبيق الخوارزمية على المثال السابق 22.45\%، أما نسبة الكبس التي يوفرها ملف الخزن (wav) على المثال السابق فهي 24.9\% ثانيا: اختبار جودة الكلام المسترجع: يمكن معرفة جودة الكلام الناتج من تطبيق الخوارزمية المقترحة بالاعتمـاد على المقاييس

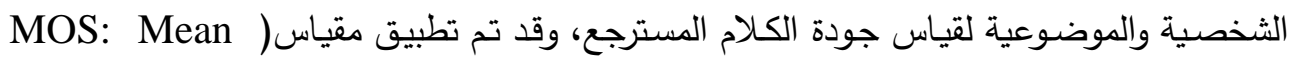
Opinion Score

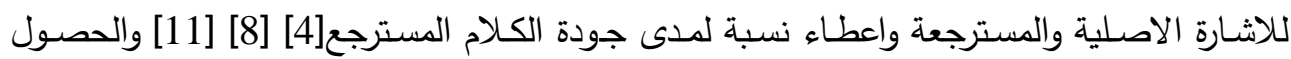
على النتائج الموضحة في الثكل (11):

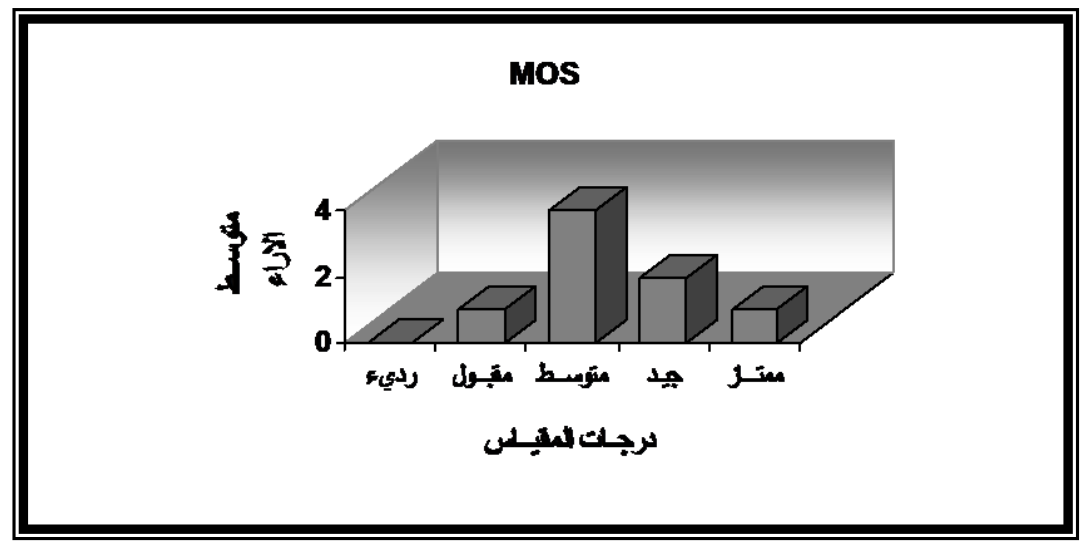

الشكل(11): قياس درجة جودة الإثارة المسترجعة باستخدام مقياس MOS

أمسا عند استخدام طريقة الاختبار الموضوعي لقياس جودة الكلام المسترجع من اعتمـاد المثال السابق فإن نتيجة حساب (SEGSNR :Signal to Noise Ratio) (4) تكون مساوية •ـ 
ومن خلال تطبيق الخوارزمية المقترحة على عدد من الأمثلة يمكن ملاحظة النتائج التي

حقتها الخوارزمية المقترحة مقارنة مع ملف الصوت(wav) التي يوضحها الجدول(3):

الجدول(3): النتائج التي حققتها خوارزمية الكبس المقترحة

\begin{tabular}{|c|c|c|c|c|c|c|c|}
\hline \multirow{2}{*}{$\begin{array}{c}\text { مقياس } \\
\text { مقتشوه (dB) }\end{array}$} & \multicolumn{2}{|c|}{ نسبة الكبس } & \multicolumn{3}{|c|}{ حجم الملف } & \multirow{2}{*}{$\begin{array}{c}\text { مدجيل } \\
\text { (sec) }\end{array}$} & \multirow{2}{*}{ ت } \\
\hline & (.wav) & (.ssc) & .wav & بالطريقة المقترحة العبس & قبل الكبس & & \\
\hline 30.3 & $24.9 \%$ & $22.6 \%$ & 46.9 & 42.4 & 188 & 3 & 1 \\
\hline 25.07 & $24.9 \%$ & $16.6 \%$ & 78.1 & 51.7 & 312 & 5 & 2 \\
\hline 22.6 & $24.9 \%$ & $17.0 \%$ & 109 & 74.3 & 497 & 7 & 3 \\
\hline 20.8 & $24.9 \%$ & $22.6 \%$ & 156 & 141 & 625 & 10 & 4 \\
\hline 27.3 & $24.9 \%$ & $14.8 \%$ & 187 & 111 & 750 & 12 & 5 \\
\hline 25.1 & $24.9 \%$ & $14.1 \%$ & 218 & 123 & 875 & 14 & 6 \\
\hline
\end{tabular}

ويمكن إعطاء توضيح أكثر للنتائج التي تم التوصل إليها عن طريق المخططات الآتية:

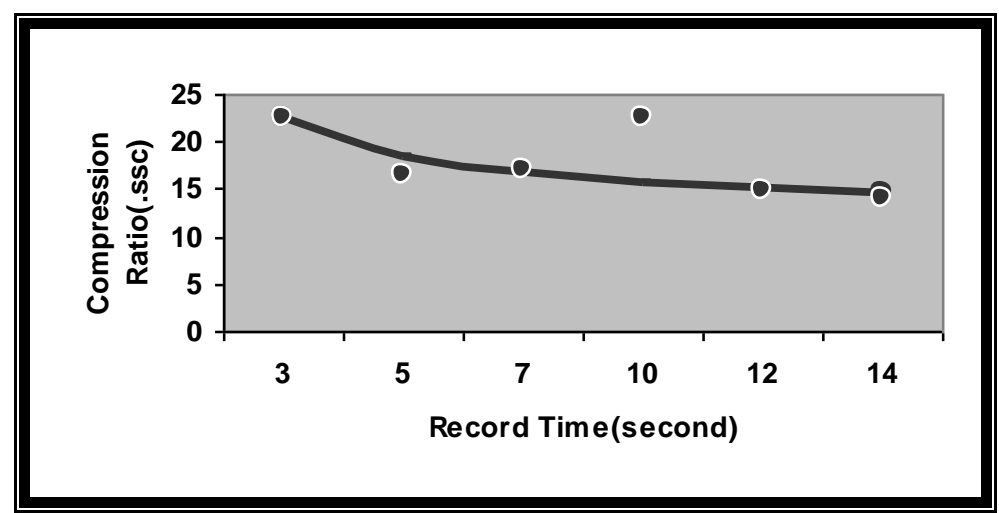

الثكل(12): نسبة الكبس المحققة وعلاقتها بالحجم 


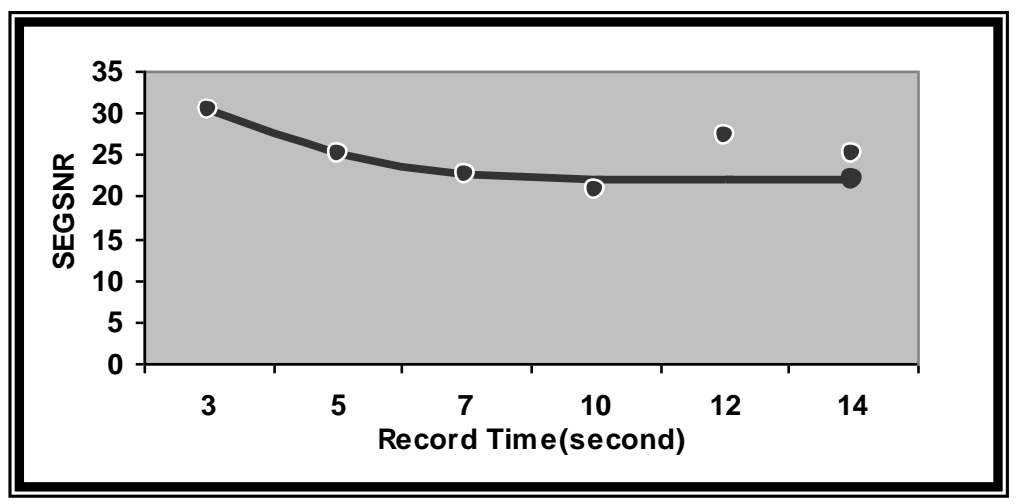

الشكل(13): نسبة التشوه وعلاقتها بحجم الملف

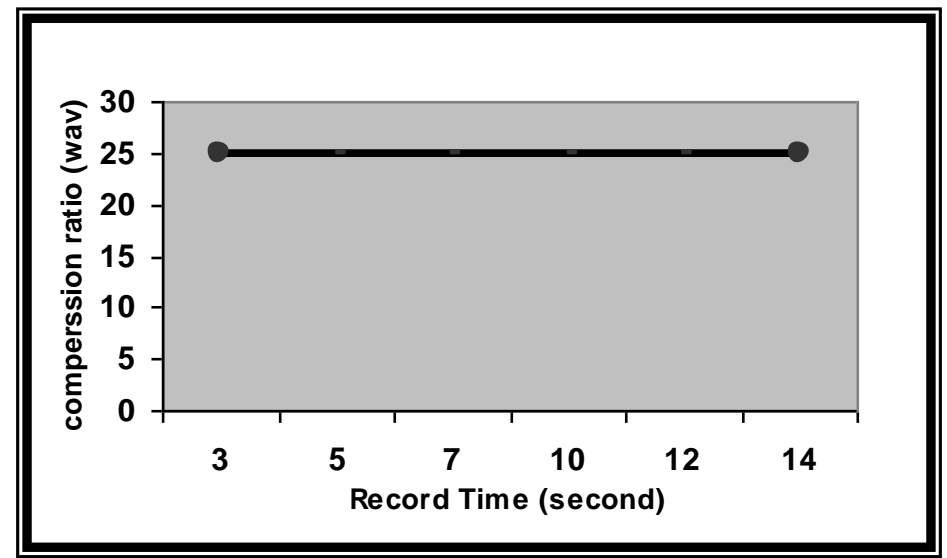

الثكل(14): نسبة الكبس في الملف (wav) وعلاقتها بالحجم

ثم طبقت الخوارزمية على مثال واحد بقيم مختلفة في مرحلة التهيئة وتم الحصول على

النتائج التي يوضحها الجدول(4) والثكل (15): 
الجدول(4) :العلاقة بين نسبة الكبس ونسبة التشوه المحققتين

\begin{tabular}{|c|c|c|}
\hline $\operatorname{SEGSNR}(d B)$ & نسبة الكبس(\%) & ت \\
\hline 28.03 & 12.24 & أولاً \\
\hline 29.72 & 15.72 & ثانياً \\
\hline 29.43 & 20.1 & ثالثاً \\
\hline 30.42 & 22.03 & رابعاً \\
\hline 30.42 & 23.42 & خامساً \\
\hline 30.63 & 29.19 & سادساً \\
\hline 31.79 & 59.14 & سابعاً \\
\hline
\end{tabular}

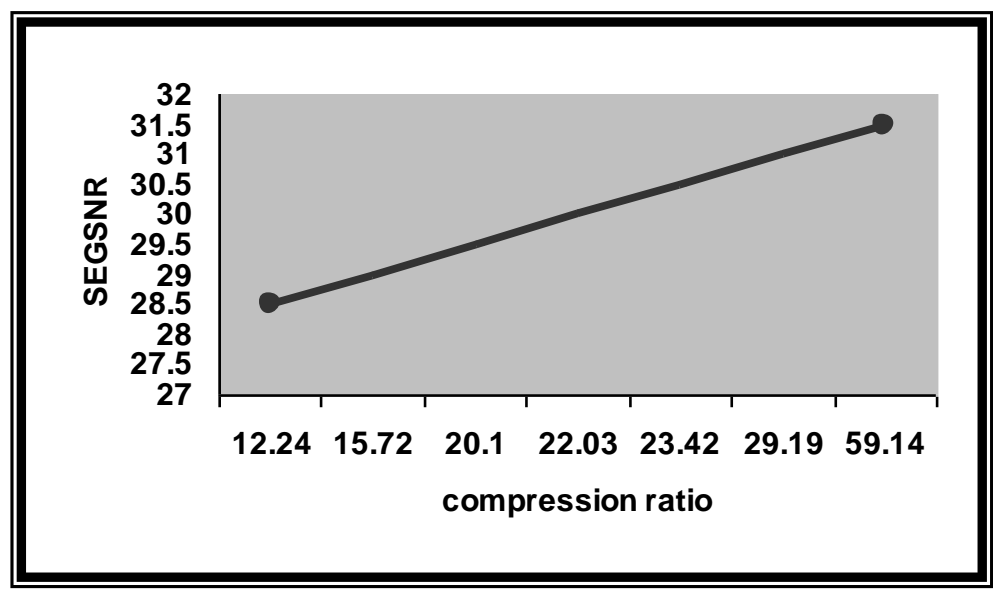

الشكل (15): العلاقة بين نسبة الكبس ونسبة التشوه المحققتين

وعند دراسـة المنحنيـات المــكورة في الأشكال (12)، (13)، (14) و (15) فضـلاً عـن

الجدولين (3) و (4) يتضـح أن الخوارزميـة المقترحة قد أعطت نتائج جيدة جداً، إذ تبين أن نسبة الذبة الكبس تؤول إلى الاستقرار عندما يبدأ حجم الملف بالاستقرار مما يجعل منها ذات فائدة كبيرة مـع الملفات ذات الأحجام الكبيرة التي تكون الحاجة ملحة إلى كبسها، فقد طبقت الخوارزمية المقترحة على أحجام مختلفة تراوحت بين KB188 إلى 
كذلك لوحظ من النتائج المبينة أن نسبة الكبس ذات علاقة خطية مع نسبة التشوه، وكون ميل تلك العلاقة قليل جداً إذ أن نسبة التشوه تزداد بمقدار قليل قد لا يصل إلى ثلث قيمة الزيادة

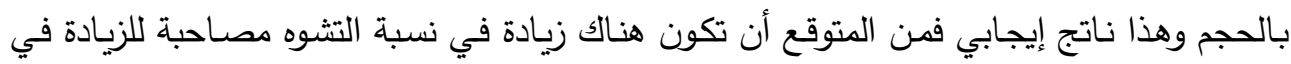

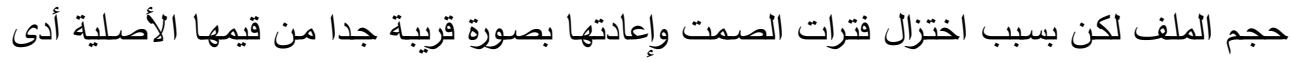
إلى ضغط نسبة التشوه وجعلها لا تتسارع تسارعاً عالياً مع زيادة حجم الملف مما يعطي دفعاً إيجابياً على النتائج العملية التي تم الحصول عليها عند تطبيق الخوارزمية.

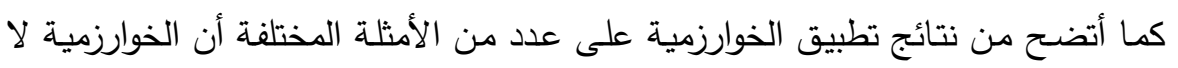
تتأثر بصفة المتكلم أو نوع الكلام وصيغته المعتمدة في التطبيقات العملية للخوارزمية، إذ انتخبت التبات التئه نماذج مختلفة من الأصوات لرجل وامرأة وطفل فضلا عن اختلاف أساليب إلقاء الكلام التي تباينت

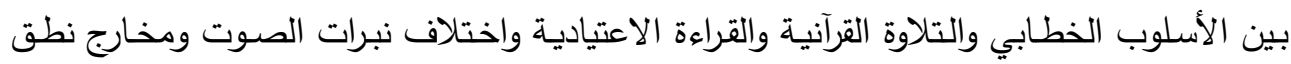
الحروف، ولم يسجل على الخوارزمية أي اختلاف في نسبة الكبس أو نسبة التشوه مع اختلاف نوع

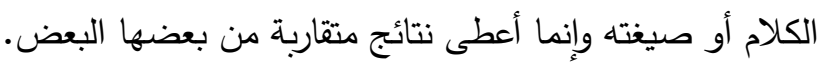
8- الاستنتاجات: • من خلال التطبيق العملي للخوارزمية المقترحة في هذا البحث تم التوصل إلى الاستتتاجات الآتية • اعتماد فكرة استخلاص خواص الكلام لغرض كبسه أعطت نتائج كبس عالية على الرغم من وجود تشوه مقبول نوعاً ما عند كبس كلام لأغراض التراسل. • إن الخوارزمية قد طرقت الباب على أسلوب غير تقليدي لأجل كبس إشارة الكلام المسموع. • إن الخوارزمية المقترحة ذات إستقرارية مع الملفات الكبيرة الحجم. • أعطت الخوارزمية المقترحة نتائج إيجابية من حيث نسبة التشوه عند تغيير حجم الملف المكبوس. • لقد حافظت الخوارزمية المقترحة على نسبة عالية جدا من الكلام، إذ أن النماذج والفترات التي يتم

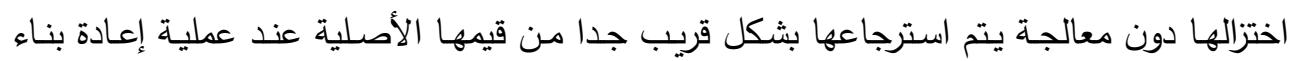


• من النقاط المهمة التي أعطتها الخوارزمية المقترحة سرية الكلام المرسل أو المخزون إذ يكون من الصعب اكتشاف ان محتويات الملف تعود إلى كلام مسموع، عليه من الممكن تطويرها لكي تعتمد

كإحدى وسائل سرية المعلومات. • أضافت الخوارزمية المقترحة أسلوباً جديداً إلى أساليب خزن إثارة الكلام بخزن معاملات مواءمة منحني الإشارة فضلا عن الطرائق التقليدية المعروفة.

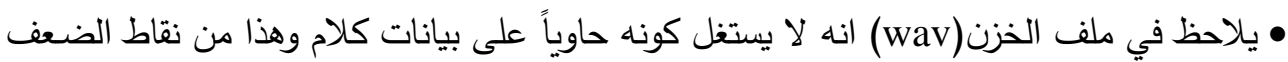
التي تسجل على الملف.

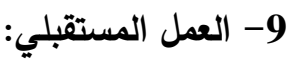
فيما يأتي بعض المقترحات للعمل المستقبلي الذي يمكن أن يبنى على ما تم اقتراحه وبناؤه ومن ثم تطبيقه : • إن الخوارزمية المقترحة تقتح باباً يمكن استغلاله لغرض تشفير الكلام المسموع والحفاظ على سرية المعلومات التي يحتويها ذلك الملف المكبوس.

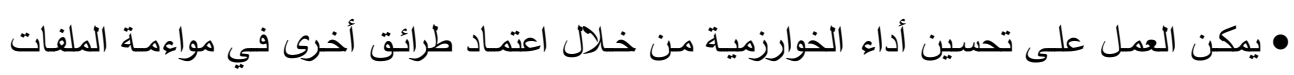
لأجل الحصـول على معادلات رياضية قريبة جدا من العلاقـة الرياضية التي تمثل إشـارة الكلام الأصلية. • العمل على تطوير الوسائل الديناميكيـة الخاصـة بتحديد واكتثـاف فترة الصــت للحصول على الصى

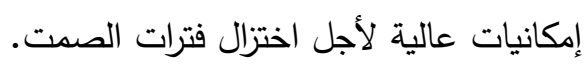
• العمل على تحسين نسبة الكبس من خلال إيجاد النسبة المثالية للتقطيع وذلك بدراسة ملفات ذات مواصفات مختلفة. 


$$
\begin{aligned}
& \text { المصادر } \\
& \text { الحديدي، إبراهيم احمد صـالح احمد (2003) "كبس الصور الرقمية باستخدام تحويلات }
\end{aligned}
$$

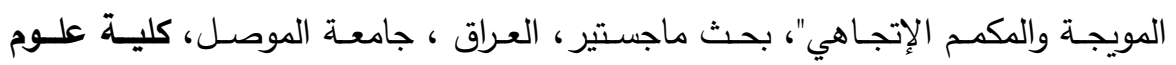

$$
\begin{aligned}
& \text { الحاسبات والرياضيات. }
\end{aligned}
$$

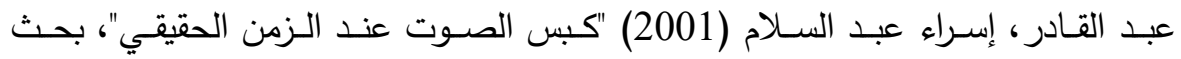

$$
\begin{aligned}
& \text { ماجستير، العراق ، جامعة الموصل، كلية علوم الحاسبات والرياضيات، . }
\end{aligned}
$$

[3] Brooks, David W.; Carr, Adam and Edkins, Keith (2004) "data Compression", Boston, U.S.A., Wikipedia the Free Encyclopedia, GNU Free Documentation License, http://en.wikipedia.org/ wiki/

[4] Hanzo, Lajos; Somerville, F. and Woodard, Jason P. (2001) "Voice Compression and Communications", IEEE press, Wiley Inter science, NY 10016-5997.

[5] Kientzle, Tim (1997) programmer's Guide to Sound, Addison_ Wesley Developers Press, ISBN 0-201-41972-6.

[6] Kivimaki, Jukka (2000) "Very low bit rate speech coding using speech recognition, analysis and synthesis", M. Sc. Thesis, Tampere university of technology, Department of information Technology.

[7] Lee, Jong-hwan ; Park, Hyung Min and Jung, Ho-Young (2002) "Feature Extraction Using Independent Component Analysis", Korea, Brain Science Research Center (BSRC). http://bsrc. kaist.ackr./braintech/image/reports/1-year/eng0101a01/eng0101a0114 .htm.

[8] Liesenborgs, Jori (2000) "Voice over IP in networked virtual environments", Ph.D. Thesis, Belgium, Limburgs University Centrum, Limburgs, May.

[9] Schroeder, Manfred R. (1999) "Speech Processing", Germany, University of Gottingen, IOS press. http://www.cs.umb.edu/ asi/ multimedia /papers/Schroeder .pdf. 
[10] Skowronski, Mark D. and G. Harris, John (2003) "Improving The Filter Bank Of Classic Speech Feature Extraction Algorithm", Bangkok, Thailand, IEEE Intl Symposium on Circuits and Systems, Vol. IV, PP. 281-284, May 25 - 28, 2003, ISBN: 0-78037761-31.

[11] Stein, Jonathan Y. (2000) Digital Signal Processing, John Wiley \& Sons, inc., USA. 